\title{
Echec de l'utilisation des produits contraceptifs chez les femmes de deux districts sanitaires en Côte d'Ivoire
}

\author{
Adon Kouadio Patrick ${ }^{1}$ \\ Maître de Conférences, Institut d'Ethno-Sociologie (IES) \\ Université Félix Houphouët-Boigny d'Abidjan, \\ E-mail : patrick_adon@yahoo.fr \\ 25 BP 104 Abidjan 25, Tel : (+225) 07685693
}

\section{Résumé}

Cet article analyse la situation reproductive des femmes sous méthodes contraceptives dans deux districts sanitaires ivoiriens. Il s'agit d'une étude rétrospective réalisée dans les services de planification familiale (SPF) et de maternité des districts sanitaires d'Adzopé et d'Agboville dans le sud-est de la Côte d'lvoire. La méthodologie de collecte des données a consisté à l'analyse de (150) dossiers de femmes fréquentant les SPF et l'exploitation de rapports d'activités des centres de santé. Ensuite, des entretiens ont été effectués, à l'aide d'un guide, avec les sages-femmes et les femmes enceintes. Les résultats établissent une faible couverture contraceptive (2 à $5 \%)$ et le recours fréquent à la contraception orale ; l'échec de la contraception a été traduit par l'abandon des consultations prénatales (34\% à Agboville et $49 \%$ à Adzopé), les accouchements à risques effectués à domicile (19\% à Agboville et $18 \%$ à Adzopé) ainsi que les avortements spontanés (58 cas à Agboville et 240 cas à Adzopé) et provoqués (69\% à Agboville et 146 cas à Adzopé) avec une augmentation (+ $63 \%$ ) à Adzopé de 200 I à 2002 chez les utilisatrices de contraceptifs des districts sanitaires.

Mots clés: Santé de la reproduction ; Echec de contraception ; Femmes ; Côte d'lvoire.

\begin{abstract}
This paper analyzes the situation of women in reproductive contraceptive methods in two Ivorian health districts. This is a retrospective study in family planning services (FPS) and maternity health districts Adzopé and Agboville in south-eastern Côte d'Ivoire. The methodology of data collection was to analyze (I50) records women attending FPS and operating activity reports of health centers. Second, interviews were conducted with the help of a guide, with midwives and pregnant women. The results establish a low contraceptive coverage $(2-5 \%)$ and the frequent use of oral contraceptives, the contraceptive failure was translated by the abandonment of antenatal care (34\% and 49\% Agboville Adzopé) unsafe childbirth performed at home (19\% to 18\% and Agboville Adzopé) and spontaneous abortions (58 cases and 240 cases Agboville Adzopé) and induced (69\% to I 46 cases and Agboville Adzopé) with an increase (+ 63\%) in Adzopé from 200 I to 2002 in users of contraceptive health districts.
\end{abstract}

Keywords: Reproductive Health, failed contraception, women, Côte d'Ivoire

\footnotetext{
' L'auteur remercie chaleureusement le rédacteur en chef de la revue APS d'avoir fait diligence pour le retour d'instruction de cet article soumis depuis juillet 2013 au comité de rédaction de African Population Studies (APS). J'adresse également mes remerciements au lecteur anonyme de cet article pour ses commentaires qui ont contribué à améliorer la première version de ce texte. II est vrai, cependant, que je reste seul responsable des positions théoriques prises et des données contenues dans cet article.
} 


\section{Introduction}

La Côte d'lvoire s'est dotée depuis 1999 d'un programme national de santé de la reproduction/planification familiale (SR/PF) (Anoh, Fassassi, et Vimard, 2004). La santé des femmes ivoiriennes en âges de reproduction est, en effet, étroitement liée à la sexualité qui rythme les attitudes et les comportements sociaux des femmes dans divers domaines de la vie domestique et familiale. L'utilisation de méthodes contraceptives peut alors favoriser un meilleur état de bien-être chez les femmes ivoiriennes en âge de reproduction surtout que le nombre de décès maternel $(70 \%)$ consécutifs à des avortements provoqués est élevé (OMS, 1997 : 30). Ainsi, le taux d'utilisation de contraception moderne reste faible (15\%) en Côte d'lvoire (OMS, 1997 : 39) en raison de facteurs culturels et sociaux (Fassassi, 2007), qui semblent compromettre la santé maternelle par l'incitation à des avortements provoqués clandestins, «une pratique répandue chez les femmes vivant en Côte d'lvoire avec une prévalence de 42,5\% \% (Bénié, et al. 2012:67).

Le choix d'analyser ces préoccupations dans les régions de l'Agneby-Tiassa et de la Mé, notamment dans le département d'Agboville (Agnéby-Tiassa) et les départements d'Adzopé (la Mé), se justifie par plusieurs raisons : d'abord, les ratios personnels de santé / populations dans le département d'Agboville font ressortir un (I) médecin pour 17532 habitants et une sage-femme pour I 900 femmes en âge de procréer, et un ( $\mathrm{I})$ infirmier diplômé d'état pour 4969 habitants (Hien, 2002). Ensuite, dans le département d'Adzopé par contre, il y a un (I) médecin pour 23579 habitants, une sage-femme pour 2967 femmes en âge de procréer et un (I) infirmier diplômé d'état pour 5804 habitants.

Au-delà de ces constats, quels sont les types de produits contraceptifs couramment utilisés par les femmes du sud-est ivoirien ? Quels sont les effets de l'échec de l'usage de la contraception sur l'état de santé des femmes utilisatrices de méthodes contraceptives? D'un point de vue théorique, quel est le niveau des connaissances scientifiques aujourd'hui en matière d'échec d'utilisation de la contraception et qui, dans ce domaine, reste inconnu dans le contexte ivoirien ? C'est sur la base de ces questions que cet article essayera de combler le vide scientifique laisser par les travaux sociodémographiques sur la question de l'échec de la contraception débattue dans les sciences biomédicales. La Côte d'lvoire est dans cette étude comme un terrain d'observation pour répondre à ces questions de recherche.
II est alors nécessaire d'évaluer les effets de l'échec des méthodes contraceptives chez les utilisatrices de contraception, en termes d'accouchements à domicile, de grossesses non désirées, d'interruption volontaire de la grossesse (IVG). Ainsi, la principale contribution de ce travail est de présenter l'échec de l'utilisation des produits contraceptifs ; cet échec se traduit par le nombre de femmes qui, malgré l'usage de la contraception, contracte une grossesse et, par conséquent fréquentent les services de consultations prénatales et d'accouchement des centres de santé.

Cet article va s'articuler autour de trois points : un bref aperçu de la méthodologie de l'étude, la présentation des résultats et enfin la discussion.

\section{Cadre théorique et méthodologie}

II porte sur les approches théoriques et les aspects méthodologiques de l'étude. D'un point de vue théorique, l'objectif général de cette étude a consisté à expliquer la santé maternelle à partir des effets induits par l'échec de l'utilisation de méthodes contraceptives. De manière spécifique, il s'agit de: i) décrire les caractéristiques sociales des utilisatrices de méthodes contraceptives; ii) identifier les différents produits contraceptifs utilisés par les femmes; iii) évaluer les effets de l'échec de la contraception, en termes de grosses contractées ou accouchements enregistrés, d'issus de grosses et de recours aux consultations prénatales.

La thèse soutenue dans ce travail est que les effets médico-sociaux de l'échec de la contraception sont empiriquement observables, d'une part, par les statistiques relatives aux consultations prénatales et aux accouchements. Ce sont ces données qui illustrent ou matérialisent le fait que les produits contraceptifs n'avaient pas atteints les effets souhaités par les utilisatrices. D'autre part, le niveau d'utilisation des produits contraceptifs par les femmes, couplé avec les issus de grossesses ont reflété l'état de santé reproductive des utilisatrices des produits contraceptifs.

Ainsi, il a été plausible d'émettre l'hypothèse que l'échec de l'usage des produits contraceptifs, par les femmes, permet d'évaluer leur état de santé et d'expliquer leur recours aux services de maternité. De fait, la faiblesse du niveau d'utilisation des produits contraceptifs a été une variable explicative des effets inattendus de la contraception chez les utilisatrices. Dans ce cas, il y a des produits contraceptifs préférentiellement utilisés par les femmes en fonction de leurs effets souhaités. Théoriquement, les caractéristiques sociales des utilisatrices influencent 
faiblement leur décision de choix de contraception mais les effets des produits semblent plus déterminants. Des données factuelles sur les produits contraceptifs, les caractéristiques des utilisatrices peuvent être obtenues dans les services de planification familiale et de maternité.

La méthodologie de l'étude a consisté à : i) l'élaboration du modèle théorique de l'étude, l'identification des objectifs, la construction de l'hypothèse et des variables, ii) et la définition d'éléments de vérification des variables, en particulier les informations à collecter, iii) l'identification des centres de santé à visiter pour la collecte des informations ainsi que l'élaboration d'outils composée de guides pour les entretiens et de grilles pour l'observation. Ainsi, six (6) formations sanitaires du département d'Adzopé et six (6) autres du département d'Agboville ont constitué le cadre de l'étude. L'étude de données a été basée sur des observations et les analyses réalisées dans les deux districts sanitaires, ont été à trois niveaux distincts mais complémentaires : i) l'analyse des services orientés vers la communauté, ii) l'analyse de 150 dossiers des utilisatrices des services de la planification familiale (PF), iii) le niveau de couverture de la consultation prénatale (CPN), et les issus de grosses et d'accouchements.

Des entretiens ont ensuite été réalisés, à l'aide d'un guide structuré autour de certains thèmes relatifs à la contraception et aux produits contraceptifs, avec des utilisatrices de produits contraceptifs. II s'agit, notamment, des femmes enceintes ayant utilisé des produits contraceptifs avant la grossesse et qui fréquentaient les services de consultations prénatales et de l'accouchement des centres de maternité et des hôpitaux de références. De même, des entretiens ont été réalisés avec des sages-femmes de la maternité et des infirmières du service de planification familiale des centres de santé d'Agboville et d'Adzopé. Ces entretiens se sont déroulés pendant les pauses de travail en les interrogeant en fonction de leur disponibilité.

Les données collectées ont porté sur les rapports d'activités de la maternité faisant ressortir les statistiques sur les consultations prénatales, les activités de planification familiale et les accouchements. Ces données portent sur la période ${ }^{2}$ de l'année 2002

\footnotetext{
${ }^{2}$ Les données de cet article se rapportent à l'année 2002 parce que cette année 2002 a été le début de la crise armée ivoirienne. Car le 19 septembre 2002, il s'est agit d'un coup d'Etat dont l'échec s'est transformé en une rébellion armée. Les faits de santé génésique que j'ai évoqués, dans
}

parce que durant cette année il y a eu une crise armée en Côte d'Ivoire. En outre, la collecte des données a été enrichie par la lecture de travaux de recherche à la bibliothèque de l'Ecole Nationale de Statistiques et d'économie Appliquée (ENSEA) d'Abidjan ainsi que des documents consultés sur Internet. Pour le traitement des données, un masque de dépouillement a permis d'analyser les fiches, de recouper les informations et de les regrouper par catégories de cibles. Le logiciel Excel a permis d'analyser et de croiser les informations issues des dossiers des utilisatrices de produits contraceptifs, avec ceux des données obtenus à partir des rapports (Hien, 2002 ; Koné, 2002 ; Koulou, 2002) d'activités des centres de santé des deux districts sanitaires.

\section{Résultats de l'étude}

L'analyse des résultats permet de faire ressortir d'abord les réponses à la question de savoir quelles sont les caractéristiques sociales (Tableau I) des utilisatrices de produits contraceptifs qui ont fréquenté les services de SR/PF des centres de santé.

cet article, en rapport avec la crise armée de 2002 en Côte d'lvoire sont mis en relief dans la discussion de l'étude. II n'y a pas eu, dans le sud-est de la Côte d'lvoire où sont localisés les départements d'Agboville et d'Adzopé, une guerre civile (entendue ici comme l'affrontement généralisé intercommunautaires ou inter-ethniques ou encore entre groupes civils) continue de 2002 à $201 \mathrm{l}$. Mais les zones à fortes tensions armées étaient l'ouest et le nord de la Côte d'lvoire. II y a eu des élections présidentielles le 3 I octobre et le 28 novembre 2010. Ainsi, au premier trimestre 20I I, il y a eu particulièrement à Abidjan une crise armée postélectorale consécutive aux résultats contestés du $2^{\text {ème }}$ tour des élections présidentielle du 28 novembre 2010 . 
Tableau I : Caractéristiques de 150 femmes utilisatrices de produits contraceptifs

\begin{tabular}{|c|c|c|c|c|c|c|c|c|c|}
\hline \multirow[b]{2}{*}{ Variables } & & \multicolumn{8}{|c|}{ Tranches d'âges } \\
\hline & & $\begin{array}{l}15- \\
19\end{array}$ & $\begin{array}{l}20- \\
24\end{array}$ & $\begin{array}{l}25- \\
29\end{array}$ & $\begin{array}{l}30- \\
34\end{array}$ & $\begin{array}{l}35- \\
39\end{array}$ & $\begin{array}{l}40- \\
44\end{array}$ & $\begin{array}{l}45 \text { et } \\
+\end{array}$ & $\begin{array}{l}\text { To- } \\
\text { tal }\end{array}$ \\
\hline \multirow{4}{*}{ Niveau d'étude } & Non scolarisé & 1 & 4 & 6 & 7 & 8 & 3 & I & 30 \\
\hline & Primaire & 8 & 14 & 9 & 8 & 7 & 4 & 2 & 52 \\
\hline & Secondaire & 12 & 17 & 10 & $\mathrm{II}$ & 5 & 5 & 2 & 62 \\
\hline & Supérieur & - & - & 2 & 2 & I & I & - & 6 \\
\hline \multirow{3}{*}{$\begin{array}{l}\text { Situation } \\
\text { matrimoniale }\end{array}$} & Veuve/divorcée & - & - & - & - & - & 1 & I & 5 \\
\hline & Célibataire & 5 & 15 & 5 & 2 & I & - & - & 59 \\
\hline & Mariée & - & 3 & 6 & 6 & 2 & 2 & I & 86 \\
\hline & Élèves/ étudiants & - & 7 & 2 & 5 & - & - & - & 30 \\
\hline Situation & Commerçante & 2 & 5 & I & 2 & I & I & - & 25 \\
\hline \multirow[t]{4}{*}{ professionnelle } & Couture/coiffeuse & 3 & 4 & I & - & - & - & I & 39 \\
\hline & Femme au foyer & - & 2 & 7 & I & 2 & 2 & I & 56 \\
\hline & Catholique & 4 & 8 & 10 & 13 & 10 & 3 & 2 & 50 \\
\hline & Protestant & I & 12 & 8 & 6 & 6 & 2 & - & 35 \\
\hline \multirow[t]{3}{*}{ Religion } & Musulman & 4 & 10 & 9 & 9 & 4 & 2 & 2 & 40 \\
\hline & Animiste & 3 & 2 & - & - & - & 2 & I & 8 \\
\hline & Autres & 9 & 3 & - & - & I & 4 & - & 17 \\
\hline Total & & 21 & 35 & 27 & 28 & 21 & 13 & 5 & 150 \\
\hline
\end{tabular}

Ces utilisatrices sont très jeunes puisque la majorité $(55,33 \%)$ à moins de 35 ans. Un nombre non négligeable d'entre les femmes sont analphabète $(30 / I 50)$, et très peu $(6 / 150)$ d'entre elles ont un niveau d'étude supérieur. Plusieurs vivent en union libre même si la plupart d'elles (86/150) sont mariées, mais elles n'ont pas une activité professionnelle stable. Mais, les utilisatrices ( $83 \%)$ ont une appartenance religieuse chrétienne et musulmane alors que la religion est traditionnellement favorable aux méthodes naturelles de contraception.

En ce qui concerne la description des différentes méthodes contraceptives, en rapport avec le troisième objectif de l'étude, d'usage chez les femmes interviewées, il y a des disparités de niveau contraceptif dans chaque district sanitaire ${ }^{3}$.

\footnotetext{
${ }^{3}$ Etant donné que l'étude s'intéresse à deux districts sanitaires en Côte d'lvoire, il aurait été intéressant de construire des tableaux et figures dans une approche comparative des deux districts. Mais, certaines données contenues dans les rapports d'activités des deux districts sont souvent incomplètes et même l'indisponibilité de certaines données rend difficile l'analyse comparative. Ce qui fait que pour l'exploitation des données dans les résultats, certaines analyses sont faites pour un seul district et non pour les deux districts. Ainsi, par exemple, une figure a été présentée sur la distribution des produits contraceptifs dans le district d'Agboville (figure 2) mais il n'y a pas son équivalent pour le district d'Adzopé. Pour ces aspects
}

Ainsi, la couverture de la contraception est très faible (2\%) dans le district sanitaire d'Agboville (figure I). Car le district sanitaire a dénombré que pour une population de 58907 femmes en âge de reproduction, il y a seulement I 123 femmes qui sont protégées par l'utilisation des produits contraceptifs. La «contraception orale» (pilule estroprogestative, pilule progestative, pilule microprogestative et autres pilules) avec les injectable sont les plus utilisés par les femmes avec un nombre plus élevé de nouveaux cas (647 pour les pilules et 473 pour les injectables) et de femmes protégées (544 pour les pilules, 464 pour les injectables et II 5 pour le DIU). de l'article, je pense qu'il faut se conférer à la discussion des résultats. 
Figure I : La couverture contraceptive dans le district sanitaire d'Agboville

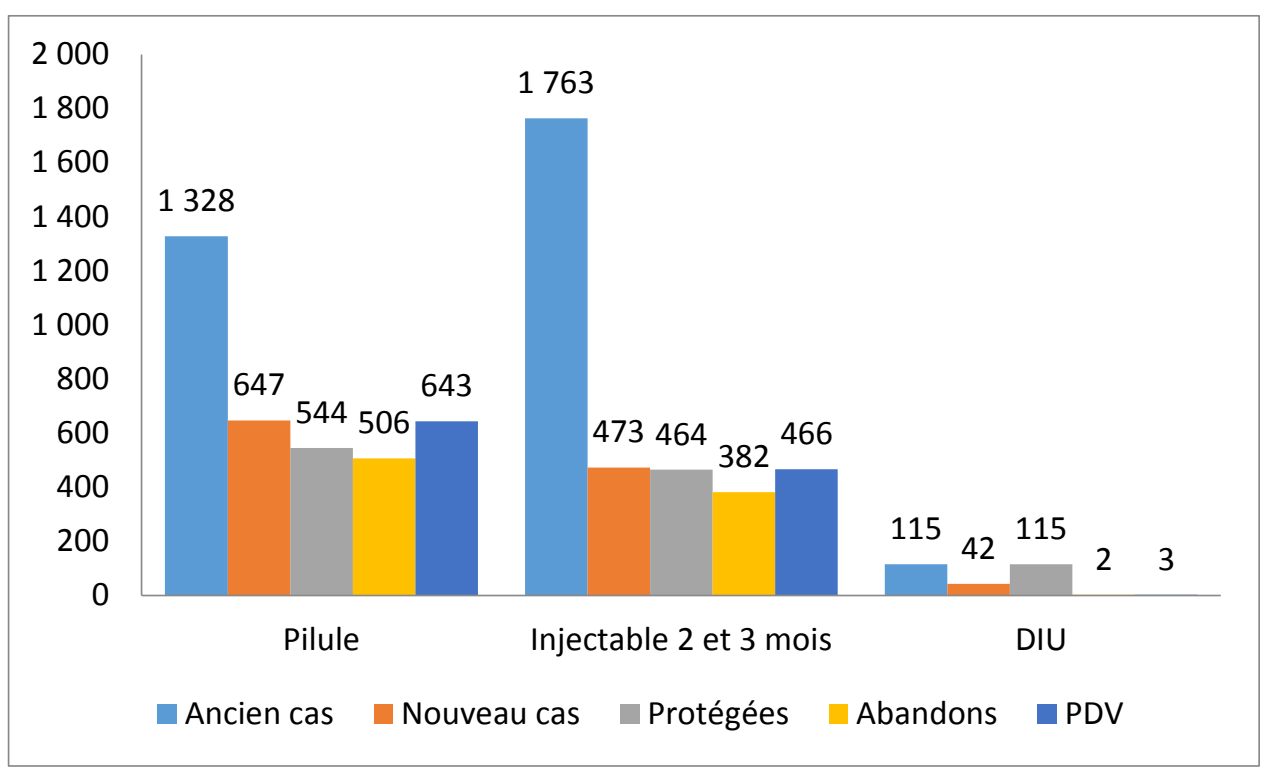

Cette faiblesse de l'utilisation des produits contraceptifs a incité les responsables du district sanitaire d'Agboville à la distribution de divers produits contraceptifs. II a été remarqué (Figure 2) que 12312 produits contraceptifs ont été distribués aux femmes du district sanitaire. Celles qui utilisaient des méthodes contraceptives ont reçu le plus souvent des pilules (9 2I2) et des injectables (2 277). Le Dispositif Intra-Utérin (35) et le condom féminin (700) et les spermicides (88) ont moins été distribués par les responsables du service de santé de la reproduction et de la planification familiale (SR/PF) du district sanitaire d'Agboville.

Figure 2 : Les unités de produits contraceptifs distribués dans le district sanitaire d'Agboville

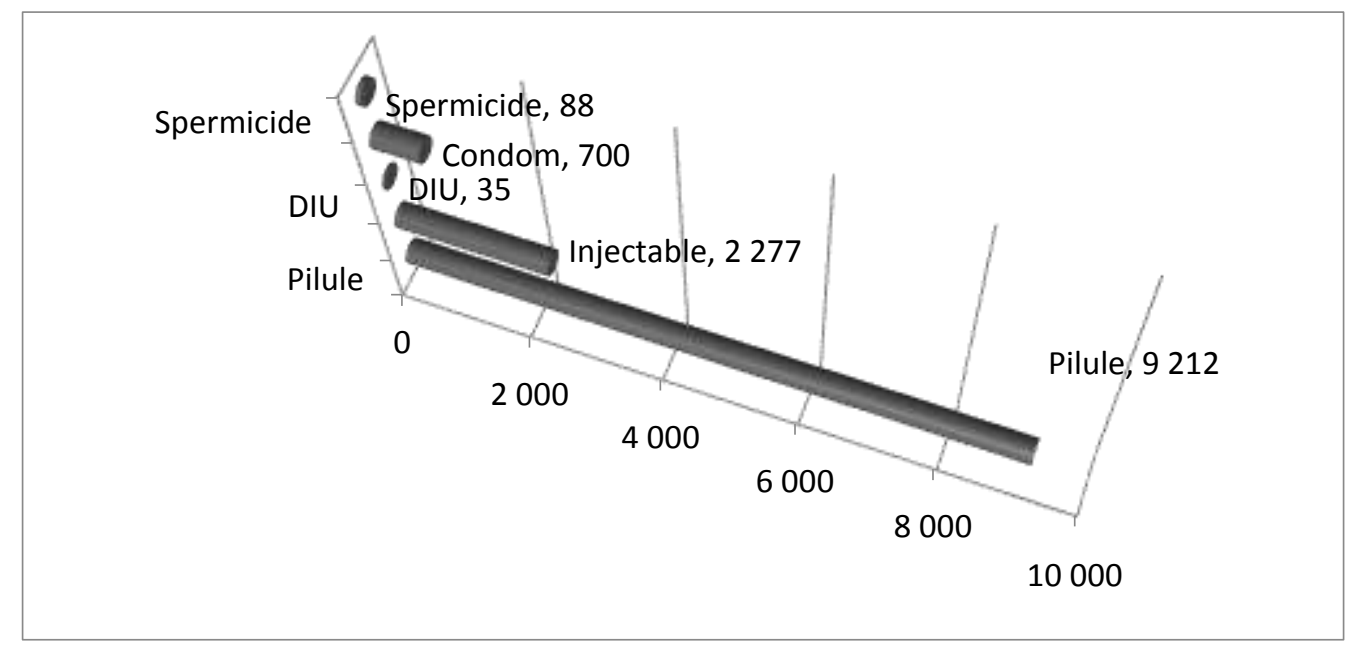

Même si les obstacles financiers (la contraception orale est à 150 francs, les injectables à 500 francs, le D.I.U est à $I 000$ francs et le néo-sampoon à 500 francs) ne sont pas susceptibles de limiter l'accès à la contraception, les responsables du programme de $\mathrm{PF} / \mathrm{SR}$ ont procédé à une distribution gratuite de produits contraceptifs. Ce soutien peut connaitre une diminution au fur et à mesure que le faible niveau de couverture contraceptive va progressive- ment commencer à avoir une relative amélioration du nombre d'utilisatrice.

Des initiatives de terrain en faveur de la communauté d'Agboville ont été effectuées par les responsables du district sanitaire pour améliorer la couverture contraceptive et surmonter les obstacles à l'accessibilité de la contraception. Ces initiatives visent à favoriser la consultation médicale en matière de santé sexuelle et reproductive afin de satisfaire le besoin d'informations et de conseil des personnes 
en âge de procréer ainsi que la proximité et confidentialité des structures de SR/PF. Ainsi, les responsables du Programme National de Santé de la Reproduction et Planification Familiale ont réalisé des séances de sensibilisation auprès des élèves de cer- tains établissements scolaires (Tableau 2). Ces expériences lancées sur le terrain ont pour objectif de prévenir les grossesses non désirées, notamment chez les jeunes filles en situation scolaire.

Tableau 2 : Les activités de SR/PF pour la communauté scolaire d'Agboville

\begin{tabular}{|c|c|c|}
\hline Établissements scolaires & Dates & Thème de communication \\
\hline Lycée Moderne d'Agboville & $13 / 03 / 2002$ & $\begin{array}{l}\text { Grossesse en milieu scolaire et hygiène cor- } \\
\text { porelle }\end{array}$ \\
\hline $\begin{array}{l}\text { Centre d'Education Technique Féminin } \\
\text { d'Agboville }\end{array}$ & $03 / 05 / 2002$ & Planification Familiale \\
\hline Collège Moderne d'Agboville & $08 / 05 / 2002$ & $\begin{array}{l}\text { Education sexuelle et prévention des } \\
\text { IST/SIDA en milieu scolaire }\end{array}$ \\
\hline Lycée Moderne d'Agboville & $10 / 05 / 2002$ & Conséquence de la sexualité de l'élève \\
\hline Collège Décroly Agboville & $31 / 055 / 2002$ & $\begin{array}{l}\text { Prévention des IST/SIDA et grossesses pré- } \\
\text { coces }\end{array}$ \\
\hline
\end{tabular}

Ainsi, dans le cadre de la I5éme journée de lutte contre le VIH/SIDA, le District Sanitaire d'Agboville a organisé une causerie débat le 04 décembre 2002 au foyer du Lycée Eyémon d'Agboville sur le thème : les méthodes contraceptives moyen de prévention $\mathrm{du} \mathrm{VIH} / \mathrm{SIDA}$. Le public cible a été la promotion des classes de 6ème, 5ème, 4ème et 2nd. Ces communications visaient à réduire le nombre de grossesses non désirées chez les jeunes filles et d'avortements en milieu scolaire par le renforcement de l'absence de structure de planification familiale dans les établissements scolaires en s'appuyant sur les professionnels de santé de la communauté géographiquement concernée par les activités des services de $\mathrm{SR} / \mathrm{PF}$ du district sanitaire.

Dans le district sanitaire d'Adzopé, par contre, les activités de planification familiale ont montré une couverture contraceptive de 22, $36 \%$ avec 15765 femmes protégées par la contraception sur une population de 70499 femmes en âge de reproduction.

Les femmes sous méthodes contraceptives à Adzopé (figure $n^{\circ} 3$ ) ont utilisé plus souvent les pilules (4 830 pour les anciennes clientes, 604 nouvelles clientes) et les injectables de trois mois (I 686 anciennes clientes et 230 nouvelles femmes). Le dispositif intra-utérin (DIU) et l'implant ont été moins utilisés. Ensuite, le nombre de femmes protégées par les méthodes contraceptives est élevé chez celles qui utilisent les pilules $(9064)$ et chez les femmes (4 65I) qui utilisaient les injectables de trois mois. Le nombre de femmes perdues de vue (PDV) est aussi élevé chez celles qui utilisent ces méthodes (I 592 pour les pilules, et I 217 pour les injectables de 3 mois) plus faciles à utiliser que le DIU et l'implant. 
Figure 3 : La couverture contraceptive dans le district sanitaire d'Adzopé

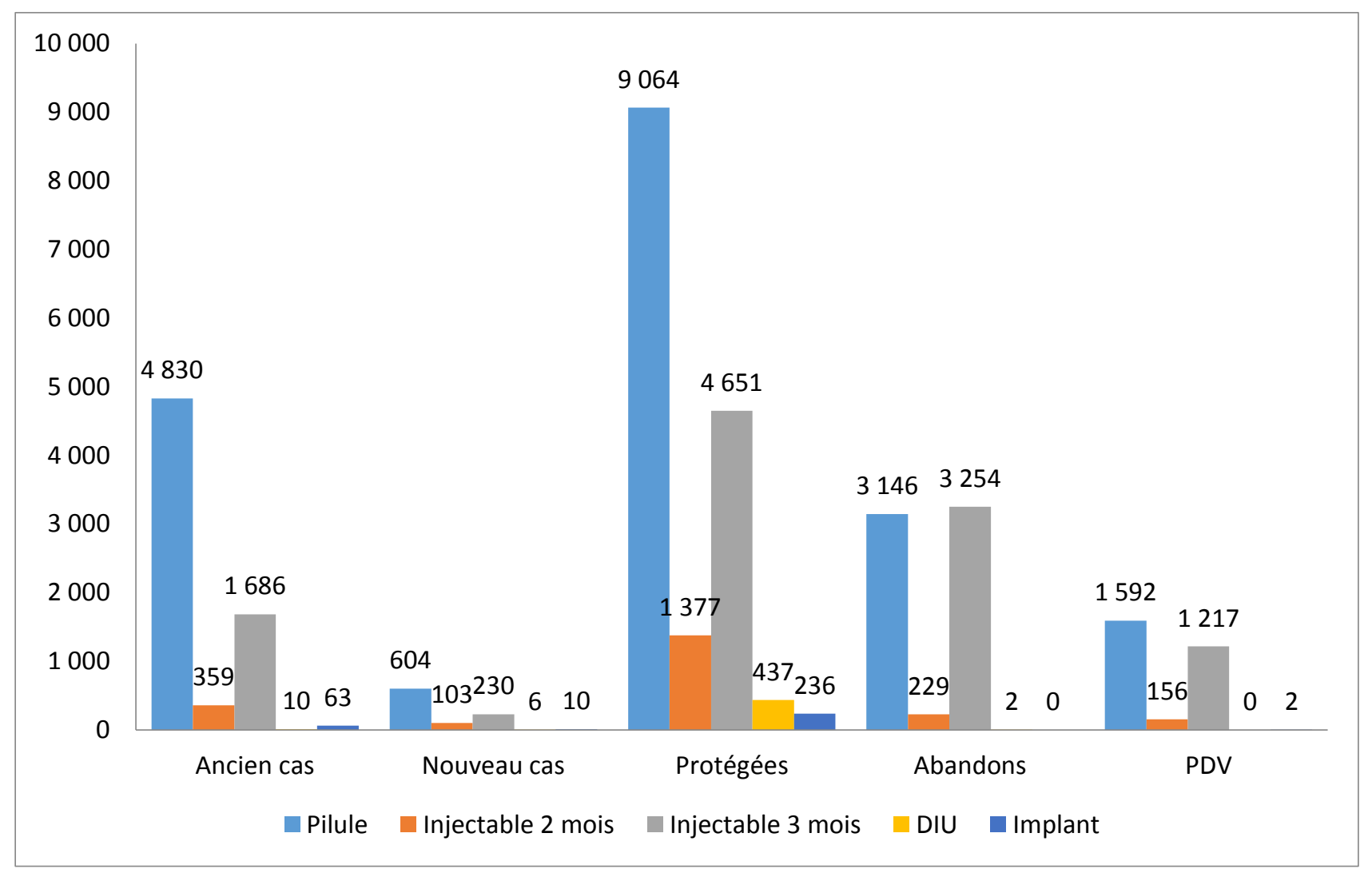

L'analyse du recours aux consultations prénatales et des issus de grossesses ont fait apparaître des taux d'échecs non négligeables de la contraception. Le premier niveau de l'échec a été perçu ici par le re cours et l'abandon des consultations prénatales (CPN) par les femmes fréquentant les services de maternité des établissements sanitaires (Tableau 3 et Tableau 4$)^{4}$.

${ }^{4}$ Dans les Tableaux 3 et 4 , l'étude se limite à $3 \mathrm{CPN}$ parce que la plupart des femmes enceintes, selon les sagesfemmes, ne vienne pas nombreuse à la $4^{\text {ème }}$ consultation prénatale alors que l'OMS recommande au minimum 4 CPN pendant la grossesse; si bien que les statistiques relatives à la $4{ }^{\text {ème }} \mathrm{CPN}$ sont insignifiantes dans les rapports d'activité des deux districts. Ainsi, dans le tableau 4 d'Agboville par exemple, les données de CPN 2 sont inexistantes (parce les statistiques incomplètes ne permettent de faire des agrégations) alors que ce tableau est l'équivalent du tableau 3 pour le district d'Adzopé. II existe des disparités dans les deux districts. 
Tableau 3 : Recours aux consultations prénatales par les femmes du district d'Agboville

\begin{tabular}{|c|c|c|c|c|c|c|c|c|c|}
\hline \multicolumn{2}{|c|}{ Aires sanitaires } & \multirow{2}{*}{$\begin{array}{l}\text { Grossesses } \\
\text { attendues } \\
-\end{array}$} & CPN I & \multicolumn{2}{|l|}{ CPN 2} & \multicolumn{2}{|l|}{ CPN 3} & $\begin{array}{l}\text { Couverture } \\
\text { en CPN } 3 \\
(\%)\end{array}$ & \multirow[t]{2}{*}{$\begin{array}{l}\text { Grossesses non pré- } \\
\text { vues = Taux. } \\
\text { d'Abandon de CPN } \\
(\%) \\
\end{array}$} \\
\hline & $\mathrm{CHR}$ & & 194 & & 154 & & 130 & - & \\
\hline \multirow[t]{2}{*}{ Agboville } & $\begin{array}{l}\text { Autres } \\
\text { FS }\end{array}$ & - & 2908 & 2280 & & 2159 & & - & 26 \\
\hline & Total & 6528 & 3102 & 2434 & & 2289 & & 35 & 26 \\
\hline \multicolumn{2}{|c|}{ CSU Azaguié } & 1049 & 967 & & 760 & & 426 & 41 & 56 \\
\hline \multicolumn{2}{|l|}{ MR Cechi } & 643 & 502 & & 359 & & 192 & 30 & 62 \\
\hline \multicolumn{2}{|c|}{ CSR Grand- Morié } & 1688 & 1210 & 1183 & & 1085 & & 64 & 10 \\
\hline \multicolumn{2}{|c|}{ CSR Oress-krobou } & 1400 & $13 \mid 4$ & 1032 & & & 920 & 66 & 30 \\
\hline \multicolumn{2}{|c|}{ CSU Rubino } & 1855 & 893 & & 498 & & 336 & 18 & 62 \\
\hline \multicolumn{2}{|l|}{ Total } & 13163 & 7988 & 6266 & & 5248 & & 40 & 34 \\
\hline
\end{tabular}

Dans le district d'Agboville, le taux d'abandon des consultations prénatales (CPN) était estimé à $34 \%$ et le taux de couverture en CPN 3 de $40 \%$ (Tableau 3).

Tableau 4 : Recours aux consultations prénatales des femmes du district d'Adzopé

\begin{tabular}{|c|c|c|c|c|c|}
\hline Aires sanitaires & $\begin{array}{l}\text { Grossesses } \\
\text { attendues }\end{array}$ & CPN I & CPN 3 & $\begin{array}{l}\text { Couverture } \\
\text { en CPN } 3 \text { (\%) }\end{array}$ & $\begin{array}{l}\text { Grossesses non prévues } \\
=\text { Taux. d'Abandon de } \\
\text { CPN (\%) }\end{array}$ \\
\hline Adzopé & 4040 & 2113 & 1015 & 25 & 52 \\
\hline Agou & 1842 & 288 & 797 & 43 & 38 \\
\hline Akoupé & 5379 & 4574 & 2423 & 45 & 47 \\
\hline Assikoi & 1014 & 581 & 415 & 41 & 29 \\
\hline Bécédi-Brignan & 609 & 429 & 231 & 38 & 46 \\
\hline Yakassé-Attobrou & 2053 & 2015 & 777 & 38 & 61 \\
\hline Total & 14937 & II 00 I & 5658 & 38 & 49 \\
\hline
\end{tabular}

Dans le district d'Adzopé, le taux d'abandon des CPN était estimé à $49 \%$ et le taux de couverture en consultations prénatales (CPN 3) de $38 \%$ (Tableau 4).

Dans toutes les formations sanitaires des deux districts, le nombre des femmes sous méthodes contraceptives qui contractaient une grossesse non désirée correspondaient au nombre de femmes qui d'abord fréquentaient irrégulièrement les services de consultations prénatales (CPN), et qui ensuite abandonnaient les consultations prénatales (CPN).
Ensuite, les femmes ne suivaient pas régulièrement les différentes consultations prénatales quand elles ont voulu interrompre la grossesse, ou encore quand elles n'étaient pas satisfaites des CPN.

Le deuxième niveau de l'échec de la contraception a été identifié par les lieux où les femmes utilisatrices de contraception (Tableau 5 et Tableau 6) ont effectué leurs accouchements ainsi que du niveau d'assistance médicale qu'elles ont bénéficié pendant l'accouchement. 
Tableau 5 : Les lieux d'accouchements et d'assistance médicale dans le district d'Agboville

\section{Lieux d'accouchement}

\begin{tabular}{|c|c|c|c|c|c|c|c|}
\hline \multirow{2}{*}{\multicolumn{2}{|c|}{ Aires sanitaires }} & \multicolumn{2}{|c|}{ Centres de santé } & \multirow{2}{*}{$\begin{array}{l}\text { Case de San- } \\
\text { té }\end{array}$} & \multicolumn{2}{|c|}{ A domicile } & \multirow{2}{*}{ Total } \\
\hline & & Effectif & $(\%)$ & & Effectif & $(\%)$ & \\
\hline & $\mathrm{CHR}$ & 747 & - & 01 & 156 & 17 & 904 \\
\hline \multirow[t]{2}{*}{ Agboville } & Autres FS & 831 & - & 01 & 173 & 17 & 1005 \\
\hline & Total & I 578 & 83 & 02 & 329 & 17 & 1909 \\
\hline \multicolumn{2}{|c|}{ CSU Azaguié } & 637 & 69 & 12 & 276 & 30 & 925 \\
\hline \multicolumn{2}{|l|}{ MR Cechi } & 289 & 90 & 00 & 31 & 10 & 320 \\
\hline \multicolumn{2}{|c|}{ CSR Grand- Morié } & 447 & 51 & 306 & 116 & 13 & 869 \\
\hline \multicolumn{2}{|c|}{ CSR Oress-krobou } & 656 & 77 & 03 & 188 & 22 & 847 \\
\hline \multicolumn{2}{|c|}{ CSU Rubino } & 280 & 78 & 27 & 51 & 14 & 358 \\
\hline \multicolumn{2}{|l|}{ Total } & 3887 & 74 & 350 & 991 & 19 & 5228 \\
\hline
\end{tabular}

Dans le district d'Agboville (Tableau 5), $19 \%$ des femmes ont accouché à domicile et 350 accouchements, réalisés par des accoucheuses traditionnelles, ont été effectués dans des cases de santé ; et le nombre de femme ayant bénéficié d'une assistance médicale dans un établissement sanitaire du district sanitaire était acceptable avec un taux d'accouchement assisté de $74 \%$; le taux d'accouchement assisté a été le plus élevé ( $90 \%)$ à la maternité rural de Cechi.

Tableau 6 : Les lieux d'accouchements et d'assistance médicale dans le district d'Adzopé

\section{Lieux de l'accouchement}

\begin{tabular}{|c|c|c|c|c|c|}
\hline \multirow{2}{*}{ Aires sanitaires } & \multicolumn{2}{|c|}{ Centres de santé } & \multirow{2}{*}{$\begin{array}{l}\text { A domicile } \\
\text { Effectif }\end{array}$} & \multirow[b]{2}{*}{$(\%)$} & \multirow[t]{2}{*}{ Total } \\
\hline & Effectif & (\%) & & & \\
\hline Adzopé & 1620 & 85 & 318 & 16 & 1938 \\
\hline Agou & 881 & 85 & 167 & 16 & I 048 \\
\hline Akoupé & 3160 & 87 & 500 & 14 & 3660 \\
\hline Assikoi & 354 & 75 & 121 & 25 & 475 \\
\hline Bécédi-Brignan & 289 & 71 & 113 & 28 & 402 \\
\hline Yakassé-Attobrou & 801 & 66 & 382 & 32 & 1183 \\
\hline Total & 7105 & 82 & 1601 & 18 & 8706 \\
\hline
\end{tabular}

II a été noté que (Tableau 6) le taux d'accouchement assisté pour le District sanitaire d'Adzopé a été satisfaisant ( $82 \%)$. Ce taux était élevé (87\%) à Akoupé. La sous-préfecture de Yakassé-Attobrou a eu un taux d'accouchement à domicile le plus élevé (32\%). Dans la même localité l'écart entre le taux d'accouchement assisté et celui d'accouchement à domicile n'étaient pas faible (34 $\%)$. L'écart entre le taux d'accouchement assisté et celui d'accouchement à domicile pour l'ensemble du District était moyen (64\%).

Le dernier niveau de l'échec de la contraception a été identifié par les issues de la grossesse des femmes (Tableau 7 et Tableau 8) ayant recours à la contraception. Ces issus de grossesses ont été exprimés en termes de naissances vivantes d'enfants mort-nés, de décès maternels et d'avortements spontanés ou provoqués. 
Tableau 7 : Les issues de grossesse dans le district sanitaire d'Agboville

\begin{tabular}{|c|c|c|c|c|c|c|}
\hline \multicolumn{2}{|c|}{ Aires sanitaires } & \multirow{2}{*}{$\begin{array}{l}\text { Naissances } \\
\text { Vivantes } \\
904\end{array}$} & \multirow{2}{*}{$\begin{array}{l}\text { Mort-nés } \\
52\end{array}$} & \multirow{2}{*}{$\begin{array}{l}\text { Décès ma- } \\
\text { ternels }\end{array}$} & \multirow{2}{*}{$\begin{array}{l}\text { Avortements } \\
\text { spontanés }\end{array}$} & \multirow{2}{*}{$\begin{array}{l}\text { Avortements } \\
\text { provoqués } \\
18\end{array}$} \\
\hline & $\mathrm{CHR}$ & & & & & \\
\hline \multirow[t]{2}{*}{ Agboville } & Autres FS & 1005 & 19 & - & 11 & 21 \\
\hline & Total & 1909 & 71 & - & 27 & 39 \\
\hline \multicolumn{2}{|c|}{ CSU Azaguié } & 925 & 15 & 0 & 08 & 06 \\
\hline \multicolumn{2}{|c|}{ MR Cechi } & 320 & 8 & - & 04 & 05 \\
\hline \multicolumn{2}{|c|}{ CSR Grand-Morié } & 869 & 7 & - & 08 & 07 \\
\hline \multicolumn{2}{|c|}{ CSR Oress-krobou } & 847 & 28 & - & 07 & 07 \\
\hline \multicolumn{2}{|c|}{ CSU Rubino } & 358 & 5 & - & 04 & 05 \\
\hline \multicolumn{2}{|l|}{ Total } & 5228 & 134 & - & 58 & 69 \\
\hline
\end{tabular}

L'analyse des dossiers, des 150 femmes utilisatrices de contraceptifs, a montré que $69 / 150$ ont pratiqué un avortement provoqué ( $46 \%$ ) alors que $58 / 150$ avortements spontanés (38\%) ont été enregistrés dans le district d'Agboville.

Tableau 8 : Les issues de grossesse dans le district sanitaire d'Adzopé

\begin{tabular}{lrcccc}
\hline Aires sanitaires & $\begin{array}{l}\text { Naissances } \\
\text { Vivantes }\end{array}$ & Mort-nés & $\begin{array}{l}\text { Décès } \\
\text { Maternels }\end{array}$ & $\begin{array}{l}\text { Avortements } \\
\text { spontanés }\end{array}$ & $\begin{array}{l}\text { Avortements pro- } \\
\text { voqués }\end{array}$ \\
\hline Adzopé & I 938 & 70 & 5 & 92 & 85 \\
Agou & I 048 & ND & 2 & 20 & 18 \\
Akoupé & 3660 & 61 & 0 & 85 & 27 \\
Assikoi & 475 & ND & 0 & 10 & 04 \\
Bécédi-Brignan & 402 & ND & 0 & 8 & 03 \\
Yakassé-Attobrou & I I83 & ND & 0 & 25 & 09 \\
\hline Total & $\mathbf{8 7 0 6}$ & I3 I & $\mathbf{7}$ & $\mathbf{2 4 0}$ & I46 \\
\hline
\end{tabular}

ND $=$ Non Déclaré

Au niveau du district d'Adzopé, il a été enregistré sept (7) cas de décès maternels. Dans les formations sanitaires, malgré le taux important d'accouchement assisté ( $85 \%$ ) à Adzopé et Agou (Tableau 6), il a été constaté le recours des femmes aux avortements spontanés et provoqués, qui avaient considérablement augmenté (+63\%) à Adzopé de 2001 à 2002 (Tableau 9) marqué par une baisse de (- 18,22\%) des consultations prénatales.
Le tableau 9 fait un focus sur les activités de santé génésique du district d'Adzopé parce que l'hôpital est un centre de référence en matière de santé de la reproduction et d'activités de planification familiale sont plus intenses par rapport au département d'Agboville qui a un Centre Hospitalier Régional avec des activités communautaires d'un niveau moindre par rapport à Adzopé. Le district sanitaire d'Agboville cordonne les activités de SR/PF de tous les centres de santé de y compris celui d'Adzopé. 
Tableau 9 : Les données spécifiques à l'hôpital général d’Adzopé

\begin{tabular}{|c|c|c|c|c|c|}
\hline \multirow{2}{*}{\multicolumn{2}{|c|}{ Activités sanitaires }} & \multirow{2}{*}{2001} & \multirow{3}{*}{$\begin{array}{r}2002 \\
1746\end{array}$} & \multicolumn{2}{|c|}{ Ecart } \\
\hline & & & & VA & VR (\%) \\
\hline \multicolumn{2}{|c|}{ Consultations prénatales } & 2135 & & -389 & $-18,22$ \\
\hline \multicolumn{2}{|c|}{ Avortements provoqués (IVG) } & 52 & 85 & +33 & $+63,46$ \\
\hline \multicolumn{2}{|c|}{ Avortements spontanés } & 102 & 92 & -10 & $-9,80$ \\
\hline & à la maternité & 1367 & 1166 & -201 & $-7,20$ \\
\hline \multirow[t]{5}{*}{ Accouchements } & à domicile & 224 & 240 & +16 & $+7,14$ \\
\hline & Total accouchement & 1591 & 1406 & -185 & $-12,20$ \\
\hline & Nombre de femmes protégées & 5972 & 6249 & +277 & $+4,64$ \\
\hline & Abandons & 145 & 186 & +41 & $+28,28$ \\
\hline & Nombre de perdus de vus & 661 & 608 & -53 & $-8,02$ \\
\hline \multirow[t]{3}{*}{$\begin{array}{l}\text { Planification } \\
\text { Familial }\end{array}$} & $\begin{array}{l}\text { Prévalence de la planification } \\
\text { familiale }\end{array}$ & 40,70 & 46,61 & $+5,91$ & $+14,52$ \\
\hline & $\begin{array}{l}\text { Taux de nouvelles accepta- } \\
\text { trices de PF }\end{array}$ & 3,5 & 2,80 & $-0,7$ & -20 \\
\hline & $\begin{array}{l}\text { Taux d'abandon de la contra- } \\
\text { ception }\end{array}$ & 2,43 & 2,98 & $+0,55$ & $+22,63$ \\
\hline
\end{tabular}

Les activités de Planification Familiale (PF) étaient en diminution à Adzopé. Car, le nombre de séance s'élève à deux (2) avec 79 participants. De même la prévalence de la PF était insuffisant $(46,61 \%$ en 2002), et le taux de nouvelles acceptatrices de produits contraceptifs de PF a été très faible $(3,5 \%$ en 200 I et $2,80 \%$ en 2002$)$ et le taux d'abandon $(2,98$ $\%)$ de la contraception n'était pas négligeable.

\section{Discussion des résultats de l'étude}

II s'agit des limites de l'étude et de l'enrichissement des résultats obtenus par d'autres travaux publiés sur la question de l'échec de la contraception. Les limites théorique et méthodologique de l'étude portent sur deux niveaux: les aspects théoriques et méthodologiques, et la fiabilité des résultats.

Au niveau des aspects théoriques et de la méthodologie, le but de cet article, qui était d'expliquer la santé des femmes utilisatrices de contraceptifs à partir des effets induits par l'échec de l'utilisation de méthodes contraceptives, a été atteint, de même que les objectifs spécifiques : l'identification des caractéristiques des utilisatrices de contraception et les produits contraceptifs couramment utilisées ; il a été aisé de procéder à une évaluation quantitative des effets de l'échec de la contraception. Cependant, les aspects qualitatifs de l'étude (discours des utilisatrices de contraception, et leurs perceptions psychosociales des effets des produits contraceptifs) qui n'ont pas été élucidé dans cet article, nécessitent d'être le plus approfondis. La thèse soutenue dans ce travail a démontré que les effets médico-sociaux de l'échec de la contraception étaient empiriquement observables d'une part par les statistiques relatives aux consultations prénatales et aux accouchements. Ce sont ces données qui ont illustré ou matérialisé le fait que les produits contraceptifs n'avaient pas atteints les effets souhaités par les utilisatrices. D'autre part, le niveau d'utilisation des produits contraceptifs par les femmes, couplé avec les issus de grossesses ont traduit l'état de santé reproductive des utilisatrices de produits contraceptifs.

Au niveau de la fiabilité des résultats de l'étude, il est à noter que le nombre de centres qui fournissent les rapports mensuels était de 29 centres à Adzopé et 19 centres à Agboville. Mais l'indicateur d'appréciation de la fiabilité des données statistiques a été le taux de complétude, qui a été obtenu par le rapport du nombre de rapports d'activité parvenu sur le nombre de rapport attendus. Le taux de complétude annuel des rapports d'activité des centres de santé a été de $95 \%$ pour le district d'Adzopé. Certains rapports parvenus (60\% dans les délais) au district sanitaire des départements n'ont pas été rempli correctement parce qu'il y a des omissions dans la transcription des données chiffrées. Ensuite, des informations sanitaires n'étaient pas souvent reportées dans les rapports. Pour ces raisons, les statistiques exploitées pourraient être sous-estimées et cacheraient des réalités sociales beaucoup plus complexes. Ces faits ont été perceptibles dans les disparités départementales par le système d'information et de gestion des données dans les établissements sanitaires. 
Ainsi, l'hypothèse qui avait été énoncée n'a pas été vérifiée à partir de tests statistiques (Khi deux par exemple). Cependant, les l'observation des données statistiques collectées, relatives aux produits contraceptifs utilisés et du recours aux consultations prénatals dans les centres de santé, corrélées avec d'autres données (Rapport mensuel de l'établissement et les dossiers des utilisatrices de contraception) sur les avortements ont fourni une lecture objective des liens socialement établits entre l'utilisation des produits contraceptifs et leurs effets inattendus chez les utilisatrices. Les résultats de cette étude étaient alors propres à l'aire géographique des deux districts sanitaires. II a été aussi difficile de procéder à une généralisation des résultats à l'échelle nationale de la Côte d'lvoire. Toutefois les résultats de cette étude, qui traduisent les réalités contraceptives des deux districts sanitaires (Agboville et Adzopé), nous ont orientés vers de nouvelles pistes de recherche issues d'autres travaux de recherche.

L'apport d'autres travaux a été les études réalisé par d'autres chercheurs ivoiriens et dans les pays étrangers. En Côte d'lvoire, le recours à l'avortement provoqué semble avoir été toléré par les pouvoir public alors qu'il est officiellement proscrit jusqu'à ce jour par le code civil. Cette tolérance de la pratique de l'avortement peut s'expliquer par son impact démographique (Degrées, et al. 1999) sur la baisse de la fécondité.

Ainsi, des travaux de récents ont montré qu'en Côte d'Ivoire "Les femmes qui ont eu le plus recours à l'avortement avaient moins de 25 ans, étaient célibataires, sans niveau d'instruction et utilisaient la contraception. Plus de la moitié de ces avortements (52,I \%) ont été pratiqués à domicile par des avorteurs traditionnels ou par les femmes elles-mêmes avec des plantes ou décoctions" (Bénié, et al. 2012 : 70). Les raisons les plus évoquées par les femmes " pour justifier le recours à l'avortement étaient dominés par la crainte de la réaction des parents ( $27,7 \%)$, l'âge trop jeune pour avoir un enfant $(22,2 \%)$, le manque de ressources financières pour assurer la charge d'un enfant ( $21,3 \%)$ et la volonté de poursuivre les étude " (Bénié, et al. 2012: 70). Tous ces arguments avaient résumé l'échec de la contraception chez celles qui les utilisaient et qui s'est traduit par la contraction d'une grossesse non désirée nécessitant des avortements.

Ces avortements souvent clandestinement provoqués, ont présenté des risques pour la santé des femmes (Coulibaly, 2009) et ont favorisé une mortalité maternelle très élevé dans le district d'Agboville
(Kreman, 2004). Tout comme notre étude qui présente des disparités entre les deux districts sanitaires, une étude récente réalisé au Kenya, a montré qu'il y avait une variation interrégional ou entre district en matière d'utilisation des méthodes contraceptives (Kimani, Njeru, et Ndirangu 20I3).

Quelle solution alors face aux échecs de la contraception? II avait été proposé, en Europe, la contraception d'urgence pour palier les échecs de la contraception. Cependant, il y a plusieurs circonstances sociales qui exposent les utilisatrices de contraception au risque d'une grossesse non désirée. "Dans ces cas, où la prévention primaire que constitue la prise d'une contraception régulière a été mise en échec, la contraception dite d'urgence constitue une solution de rattrapage qui s'apparente à une forme de prévention secondaire, et permet d'éviter le recours éventuel à l'interruption volontaire de grossesse. La forme la plus connue de la contraception d'urgence est la "pilule du lendemain" " (Aubin, et al. 2009 : 37).

Le recours à l'interruption volontaire de grossesse (IVG) a continué à augmenter malgré les campagnes de sensibilisation sur l'utilisation de la contraception d'urgence. "Les mesures prises pour faciliter l'accès à la contraception d'urgence étaient explicitement motivées par la volonté de voir diminuer le nombre des grossesses non désirées notamment chez les jeunes filles et donc de prévenir les avortements. Force est de constater que cet objectif est pour le moment resté lettre morte malgré la montée en charge de la "pilule du lendemain" puisque l'on observe une stabilité globale du nombre d'IVG pratiquées avec une tendance à l'augmentation chez les jeunes " (Aubin, et al. 2009 : 45). Cependant, « l'accès libre et gratuit des mineures à la contraception d'urgence entraîne une hausse des grossesses adolescentes et une prévalence accrue des maladies sexuellement transmissibles dans cette population"(Arduin, 20II: I). En effet, les taux de grossesses non désirées chez les jeunes filles britanniques où la pilule du lendemain est fournie gratuitement en pharmacie aux adolescentes de moins de 16 ans sont plus élevés que chez celles où une prescription médicale est nécessaire.

Toutes ces études ont démontré que «les échecs de contraception restent fréquents : aujourd'hui encore une grossesse sur trois est qualifiée de "non prévue" (contre près d'une sur deux en 1975), qui donnera lieu, dans $60 \%$ des cas, à une IVG. Deux grossesses non prévues sur trois surviennent chez des femmes qui déclarent utiliser une contraception au moment de la survenue de ladite grossesse. Cette couverture contraceptive reposait dans près de la moitié des cas sur une méthode de contraception médicalisée » présentant 
une efficacité théorique très élevée (pilule et stérilet) » (Aubin, 2009 : 12).

Les nouvelles pistes de recherche futures qui vont être exploré, ont été regroupées en trois volets. D'abord, il y a les pistes de recherches socioanthropologiques sur la grossesse. La pensé génésique des groupes ethnoculturels akan en Côte d'Ivoire, dont appartiennent les districts d'Agboville et d'Adzopé, a toujours considéré la grossesse comme étant sacré et son interruption volontaire semble culturellement proscrite (Abé, 20I3). II s'agit d'interroger les représentations socioanthropologiques de la conception pour une meilleure prévention des avortements et des grossesses non prévues. Comment expliquer cette mutation des idées génésiques dans ces groupes ethnoculturels où la grossesse et la maternité sont socialement valorisées?

Cette étude s'est intéressée aux données de l'année 2002 à cause de la crise armée survenue en Cote d'Ivoire au cours de cette année. II s'agit aussi de rechercher l'impact psychosocial et génésique des crises armées (2002 et 20II) en Côte d'lvoire sur l'issu de la grossesse des femmes enceintes durant cette période de conflit. Les crises armées successives ont favorisé une situation plus élevée de vulnérabilité des femmes enceintes qui peuvent présenter plus de vulnérabilité psychologique, par l'effet des tirs d'armes et des déplacements forcés, sur l'évolution et l'issu de la grossesse pouvant abouti à un avortement spontané.

Ensuite, il s'agit des pistes de recherche médicosociales. II est impératif d'évaluer comment de trop nombreuses erreurs de diagnostics prénatals peuvent déterminer des accouchements à domicile (Benié, et al. 2009), par exemple d'un point de vue médical et social, et conduire à des interruptions de grossesse, si bien que les avortements (provoqués ou spontané) peuvent être le fait d'erreurs de diagnostics prénatals par les professionnels de la santé. II s'agit de rechercher aussi le taux de grossesse prévisible en fonction de la méthode contraceptive utilisée par les femmes, prenant en compte les causes d'échec de la méthode contraceptive (Hatcher, et al. 2013). Mais surtout, les études à approfondir vont se porter sur les taux d'avortement (provoqué ou/et spontané) prévisible chez les femmes sous méthodes contraceptives en analysant les causes contraceptives des avortements, c'est-àdire les produits contraceptifs ayant été des facteurs déterminants la rupture de la reproduction.

Enfin, «l'étude de la satisfaction des patientes envers leur contraception »(Baudry, 2010:35) réalisée en
France a montré que plus de $90 \%$ des femmes qui utilisent la contraception orale ont déclaré être satisfaite. Mais la nature de cette satisfaction n'est pas explicitée en fonction de l'efficacité souhaitée des moyens contraceptifs ou des objectifs attendus par les femmes en termes de grossesse possible et d'avortement prévisible. Cependant, l'étude du changement de produit contraceptif est une piste de recherche à explorer pour évaluer la satisfaction des femmes de produits contraceptifs utilisés et les motifs de leur choix pour un nouveau produit contraceptif. Les raisons évoquées par les utilisatrices françaises sont en rapport avec les motifs d'insatisfaction des patientes envers leur moyen contraceptif: le manque de confort $(48.3 \%)$, les troubles physiques (31.0\%) et le désir de grossesse $(6.9 \%)$ ainsi que le manque de fiabilité (13.8\%) (Baudry, 2010 : 39). Les femmes sous contraception s'exposant aux risques d'une grossesse non désirée, sont celles qui abandonnent la contraception pour des motifs de commodité, de manque de confort ou de troubles physiques sans avis ou consultation médicale.

\section{Conclusion}

La contraception met en relief la question des rapports entre la médecine de reproduction, l'inimitié sexuelle du couple et les besoins de natalité de la société. "Les programmes de planification familiale sont présentés comme un moyen de libérer les femmes d'une oppression pluri-millénaire, les grossesses non désirées, et ils ont souvent eu cette fonction. Néanmoins, leur objectif a bien souvent été purement néomalthusien: faire diminuer la fécondité, parfois à n'importe quel prix» (Gautier, 2007 : 56). La contraception a alors remplacé l'avortement qui était le recours fréquent contre la grossesse non désirée. Elle a aussi provoquée l'intrusion des professionnels de la reproduction (médecin gynéco-obstétricien et des sages-femmes) dans l'intimité sexuelle et sentimentale de la femme par la médicalisation du contrôle des naissances.

C'est dans ce contexte que se situent les activités du programme ivoirien de santé de la reproduction et planification familiale (SR/PF) qui fait partir des Sept (7) domaines d'actions prioritaires ou programmes prioritaires du PNDS (Côte d'lvoire, 2008 : 72). II faut, cependant, souligner qu'il n'y a pas de contraception idéale. Car chaque femme présente une spécificité biophysiologique et sociale spécifique de telle sorte qu'il lui est nécessaire d'avoir une contraception adaptée. C'est pourquoi, la prescription contraceptive des spécialistes de la $\mathrm{SR} / \mathrm{PF}$ est à adapter en tenant compte des variables 
morales, religieuses, du profil psychosociologique et des antécédents gynécologiques de la femme. C'est en cela que réside l'efficace d'une prescription contraceptive visant à prévenir les naissances non désirés et à garantir une meilleure santé reproductive et génésique des femmes.

La contraception est le seul moyen préventif de toutes les formes d'interruption de la grossesse qu'elle soit volontaire ou provoquée. Les paramètres de commodité, de fiabilité, d'incidence physiologique et d'efficacité clinique, en matière de prévention de la grossesse, de chaque méthode contraceptive est déjà connue par les spécialistes de la SR/PF (Stober, et Wecker., 1993). Ainsi, les situations à risque de grossesse chez des femmes sous méthodes contraceptives peuvent être considérées comme le fait, d'une part, des femmes ayant une faible connaissance des changements biophysiologiques inhérents à leur cycle menstruel et affectant leurs comportements sexuels et reproductifs. D'autre part, il y a des professionnels de la santé de la reproduction, qui communiquent insuffisamment sur les questions contraceptive avec les femmes.

Car il existe des méthodes de contraception d'une exactitude fiabilité à même de tenir en échec l'irruption d'une grossesse non désirée par une femme. L'échec de la contraception se traduit donc par les grossesses non désirées (Bajos, et al. 2002), l'abandon des consultations prénatals et les avortements clandestins à risque. Le renforcement de la promotion de l'éducation à la santé reproductive en milieu rural et scolaire, auprès des jeunes filles et des femmes va contribuer à améliorer la santé des femmes en âges de procréer. C'est pourquoi des mesures incitatives axées sur des campagnes de sensibilisation sont à réaliser (Adon, 20/2). Ces messages éducatifs pour la santé des femmes peuvent porter sur les thèmes de culturels valorisant la grossesse et la maternité ainsi que les risques de mortalité à l'issue des avortements effectués clandestinement ou ceux réalisés en milieu hospitalier.

\section{Références}

Abé, N. N. 2013. "Les fondements anthropologiques de la pensée génésique akan en Côte d'Ivoire" European Scientific Journal, Vol. 9, 5 : 5670

Adon, K. P. 2012. La santé préventive dans le sud-est ivoirien : programmes institutionnels et perspectives sociales, Sarrebruck, Éditions Universitaires Européennes, $212 \mathrm{p}$.

Anoh, A., Fassassi, R. et Vimard, P. 2004. "Politique de population et planification familiale en Côte
d'Ivoire", pp. 195-23I. In Gautier A. (dir), Les politiques de planification familiale: Cinq expériences nationales. Paris, IRD, Centre français sur la Population et le Développement, Laboratoire Population-Environnement-Développement, $246 \mathrm{p}$.

Arduin, P.-O. 20II. L'échec de la contraception d'urgence, 2 p. [en ligne],

http://www.libertepolitique.com/Actualite/Decry ptage/L-echec-de-la-contraception-d-urgence (consulté le 17/06/2013)

Aubin, C. Jourdain-Menninger, D. et Chambaud, L. 2009. La prévention des grossesses non désirées: contraception et contraception d'urgence, Paris, Inspection générale des affaires sociales, 99 p. [en ligne]

http://www.ladocumentationfrancaise.fr/var/stora ge/rapports-publics/I04000049/0000.pdf (consulté le 17/06/2013)

Bajos, N. et al. 2002, De la contraception à l'avortement : sociologie des grossesses non prévues. $\mathrm{Pa}-$ ris, INSERM, $348 \mathrm{p}$.

Baudry, É. 20I0. Les patientes sont-elles satisfaites de leur contraception ?, Thèse de doctorat de médecine, Université Paris XI, $78 \mathrm{p}$.

Benié, B. V. J. et al. 2009. "Prévalence et déterminants des accouchements à domicile dans deux quartiers précaires de la commune de Yopougon (Abidjan), Côte d'Ivoire." Santé Publique, Vol. 21, $5:$ 499-506.

Benié, B. V. J. et al. 2012. "Épidémiologie des avortements provoqués en Côte d'lvoire." Santé Publique, Vol. 24, HS : 67-76

Côte d'Ivoire, 2008. Plan National de Développement Sanitaire 2008-2012 (PNDS), Tome I: diagnostic, priorités sanitaires objectifs et stratégies, Abidjan, Ministère de la Santé, $87 \mathrm{p}$.

Coulibaly, G. L. 2009. "Connaissances, attitudes et pratiques de l'avortement provoqué clandestin en Côte d'Ivoire." Actes du XXVle congrès international de la population, Marrakech - Maroc, 27 septembre au 02 octobre 2009, 6 p.

Desgrées, D. L. A. et al. 1999. "Le recours à l'avortement provoqué à Abidjan : une cause de la baisse de la fécondité ?" Population, 54, 3 : 427446.

Fassassi, R. 2007. Facteurs de la contraception en Côte d'lvoire au tournant du siècle. Paris, CEPED, $65 \mathrm{p}$.

Gauthier, A. 2007. “ Anthropologie des persécutions genrées des femmes”, pp. 33-53. In Freedman, J. Valluy, J. Persécutions des femmes. Savoirs, mobilisations et protections. Broissieux, Edition du Croquant, Col Terra 
Guillaume, A. 1988. Santé de la reproduction en pays Akyé. Abidjan Orstom, 83 p.

Hatcher, R. A. et al, 20I3. Echec de contraception [en ligne]

http://www.piluledulendemain.com/echecs-decontraception.php (consulté le 17/06/2013)

Hien S., 2002, Rapport d'activités du district sanitaire d'Agboville, Agboville, inédit, $24 \mathrm{p}$.

Kimani, M. Njeru, M. et Ndirangu, G. 2013. "Regional variations in contraceptive use in Kenya : comparison of Nyanza, Coast and Central Provinces", African Population Studies, Vol. 27, I : 4352

Koné, F. 2002. Rapport d'activité du district sanitaire d’Adzopé, Adzopé, inédit, 60 p.
Koulou, E. A. R., 2002. Bilan de gestion 2002 et budget prévisionnel initial des ressources propres exercice 2003 de l'hôpital général d’Adzopé, Adzopé, inédit, $60 \mathrm{p}$.

Kreman, D. J. 2004. Mortalité maternelle à la maternité du CHR d'Agboville du ler Janvier 2000 au 3 I Décembre 200I, Abidjan, Université de Cocody, Thèse de médecine, $85 \mathrm{p}$.

OMS, 1997. Profil santé de la femme en Côte d'lvoire, Abidjan, Bureau OMS, $52 \mathrm{p}$.

Stober, I. H. et Wecker, Barry, H. 1993. Manuel de santé pour animateur chrétien, Londres, The Macmillan Press LTD, 242 p. 